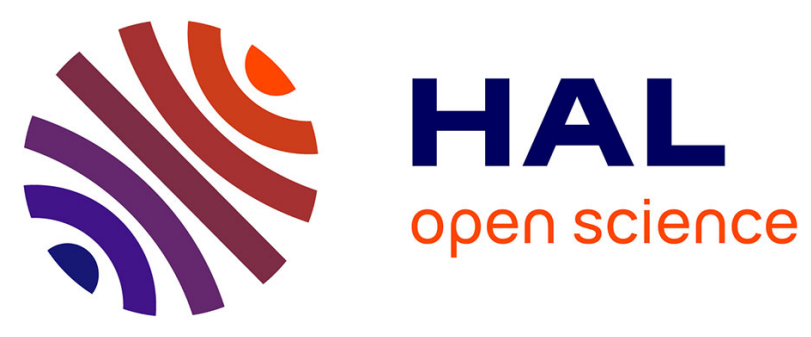

\title{
3D brain tumor segmentation in MRI using fuzzy classification, symmetry analysis and spatially constrained deformable models
}

\author{
Hassan Khotanlou, Olivier Colliot, Jamal Atif, Isabelle Bloch
}

\section{To cite this version:}

Hassan Khotanlou, Olivier Colliot, Jamal Atif, Isabelle Bloch. 3D brain tumor segmentation in MRI using fuzzy classification, symmetry analysis and spatially constrained deformable models. Fuzzy Sets and Systems, 2009, 160 (10), 10.1016/j.fss.2008.11.016 . hal-01251278

\section{HAL Id: hal-01251278 \\ https://inria.hal.science/hal-01251278}

Submitted on 5 Jan 2016

HAL is a multi-disciplinary open access archive for the deposit and dissemination of scientific research documents, whether they are published or not. The documents may come from teaching and research institutions in France or abroad, or from public or private research centers.
L'archive ouverte pluridisciplinaire HAL, est destinée au dépôt et à la diffusion de documents scientifiques de niveau recherche, publiés ou non, émanant des établissements d'enseignement et de recherche français ou étrangers, des laboratoires publics ou privés.

\section{(1) (1) $\$$}

Distributed under a Creative Commons Attribution - NonCommercial - NoDerivatives 44.0 


\title{
3D Brain Tumor Segmentation in MRI Using Fuzzy Classification, Symmetry Analysis and Spatially Constrained Deformable Models
}

\author{
Hassan Khotanlou ${ }^{\mathrm{a}}$, Olivier Colliot ${ }^{\mathrm{b}}$, Jamal Atif ${ }^{\mathrm{c}}$, \\ Isabelle Bloch ${ }^{\text {a,* }}$ \\ ${ }^{a}$ Ecole Nationale Supérieure des Télécommunications (GET - Télécom Paris) \\ Signal and Image Processing Dept, CNRS UMR 5141 LTCI, Paris, France - \\ IFR49 \\ ${ }^{\mathrm{b}}$ CNRS, UPR 640 - Cognitive Neuroscience and Brain Imaging Laboratory, \\ Université Pierre et Marie Curie - Paris 6, Hôpital de la Pitié-Salpêtrière, Paris, \\ France - IFR 49 \\ ${ }^{\mathrm{c}}$ Groupe de Recherche sur les Energies Renouvelables (GRER), Université des \\ Antilles et de la Guyane, Campus de St Denis 97300 Cayenne, France
}

\begin{abstract}
We propose a new general method for segmenting brain tumors in 3D magnetic resonance images. Our method is applicable to different types of tumors. First, the brain is segmented using a new approach, robust to the presence of tumors. Then a first tumor detection is performed, based on selecting asymmetric areas with respect to the approximate brain symmetry plane and fuzzy classification. Its result constitutes the initialization of a segmentation method based on a combination of a deformable model and spatial relations, leading to a precise segmentation of the tumors. Imprecision and variability are taken into account at all levels, using appropriate fuzzy models. The results obtained on different types of tumors have been evaluated by comparison with manual segmentations.
\end{abstract}

Key words: Brain Tumor, Segmentation, Deformable Model, Spatial Relations, Symmetry Plane.

\footnotetext{
* Corresponding author. Tel: +33 1458175 85, Fax: +331 45813794

Email addresses: hassan.khotanlou@enst.fr (Hassan Khotanlou), olivier.colliot@chups.jussieu.fr (Olivier Colliot), jamal.atif@guyane.univ-ag.fr (Jamal Atif), isabelle.bloch@enst.fr (Isabelle Bloch).
} 


\section{Introduction}

Brain tumor detection and segmentation in magnetic resonance images (MRI) is important in medical diagnosis because it provides information associated to anatomical structures as well as potential abnormal tissues necessary to treatment planning and patient follow-up. The segmentation of brain tumors can also be helpful for general modeling of pathological brains and the construction of pathological brain atlases [31]. Despite numerous efforts and promising results in the medical imaging community, accurate and reproducible segmentation and characterization of abnormalities are still a challenging and difficult task because of the variety of the possible shapes, locations and image intensities of various types of tumors. Some of them may also deform the surrounding structures or may be associated to edema or necrosis that change the image intensity around the tumor. Existing methods leave significant room for increased automation, applicability and accuracy.

The aim of this paper is to contribute to this domain, by proposing an original method, which is general enough to address a large class of tumor types.

Let us briefly summarize existing work, classically divided into region-based and contour-based methods. Most of them are usually dedicated to full enhanced tumors or specific types of tumors, and do not extent easily to more general types. In the first class, Clark et al. [2] have proposed a method for tumor segmentation using a set of rules expressed in a knowledge base and fuzzy classification, where a learning process prior to segment a set of images is necessary and which requires multi-channel images such as T1-weighted, T2-weighted and proton-density (PD). Several methods are based on statistical pattern recognition techniques. Kaus et al. [12] have proposed a method for automatic segmentation of small brain tumors using a statistical classification method and atlas registration. Moon et al. [20] have used the expectation maximization (EM) algorithm and atlas prior information. These methods fail in the case of large deformations in the brain and they also require multichannel images (T1, T2, PD and contrast enhanced images), which are not always available in clinical routine. Prastawa et al. [24] consider the tumor as outliers of the normal voxels distribution; a statistical classification based on a learning step using the atlas provides a rough segmentation and geometric and spatial constraints are then used for the final segmentation. This method does not consider large deformations of brain structures, and when such deformations occur, the use of the brain atlas may lead to incorrect learning. In [5] a method based on the combination of model-based techniques and graph-based affinity is proposed: four classes (edema, tumor, non-brain and brain matter) are first modeled in a Bayesian framework and then the tumor and edema are segmented using the segmentation weighted aggregation (SWA) method. This method also uses multi-channel MR images and needs a learning step for es- 
timating the parameters. Other atlas-based methods have been proposed, for example in [6]: after an affine registration between the atlas and the patient image, the registered atlas is seeded manually by selecting a voxel of lesion regions. Then a non-rigid deformation method at this voxel is performed with two forces: the demons force [30] outside the lesion and a prior model of tumor growth inside it. The radial growth model has been considered, which is appropriate for specific types of tumors only. The fuzzy connectedness method has been applied by Moonis et al. [21]. In this semi-automatic method, the user must select the region of the tumor. The calculation of connectedness is achieved in this region and the tumor is delineated in 3D as a fuzzy connected object containing the seed points selected by the user. Other methods dealing with multi-channel images rely on data fusion approaches. In [1] a method is proposed based on evidence theory. First the data are modeled according to an evidential parametric model (Denoeux' model, Shafer's model or Appriou's model). Then spatial information (in this case spatial neighborhood information) is used by a weighted Dempster's combination rule. Recently Dou et al. [8] have proposed a fuzzy information fusion framework for brain tumor segmentation using T1-weighted, T2-weighted and PD images. This method is sensitive to noise and needs some user interactions. A Support Vector Machine (SVM) classification method was recently applied in $[25,36]$, which needs a learning process and some user interactions.

In addition to the already mentioned limitations of each method, in general region-based methods exploit only local information for each voxel and do not incorporate global shape and boundary constraints.

In contour-based methods, Lefohn et al. [17] have proposed a semi-automatic method for tumor segmentation using level sets. The user selects the tumor region, so as to initialize a first segmentation. Based on a visual inspection of the results, he tunes the level set parameters and the segmentation process is repeated. Zhu and Yang [37] introduce an algorithm using neural networks and a deformable model. Their method processes each slice separately and is not a real 3D method. Ho et al. [9] associate level set evolution with region competition. Their algorithm uses two images (T1-weighted with and without contrast agents) and calculates a tumor probability map using classification, histogram analysis and the difference between the two images, and then this map is used as the zero level of the level set evolution. In [33] a semi-automatic method based on level sets was proposed. In this approach, the user selects a ROI and then a level set method is applied to segment the tumor.

Contour based deformable models suffer from the difficulty of determining the initial contour, tuning the parameters and leakage in ill-defined edges.

In this paper we propose a method that is a combination of region-based and contour-based paradigms. It works in 3D and on standard routine T1-weighted 
acquisitions. First of all we segment the brain to remove non-brain data (skull, fat, skin, muscle) from the image. However, in pathological cases, standard segmentation methods fail, in particular when the tumor is located very close to the brain surface. Therefore we propose an improved segmentation method, relying on the approximate symmetry plane. To provide an initial detection of the tumor we propose two methods. The first one is a fuzzy classification method that is applicable to hyper-intense tumors while the second one is based on symmetry analysis and applies to any type of tumor. In this method we first calculate the approximate symmetry plane and then symmetry analysis is performed to determine the regions that deviate from the symmetry assumption. The aim of the detection approach is to roughly locate the tumor. This does not provide an accurate estimation of its boundaries and we therefore propose a refinement step. This is achieved through a parametric deformable model constrained by spatial relations.

The paper is organized as follows. First an overview of the proposed method is presented in Section 2. Then a method to compute the approximate symmetry plane is explained in Section 3. It will be used both for brain segmentation and initial tumor detection. In Section 4 we propose a method for brain segmentation. In Section 5 two methods are presented for the initial segmentation of tumors. The segmentation refinement is presented in Section 6. Section 7 presents the results with their evaluation and some conclusions are presented in Section 8.

\section{Method overview}

The automated brain tumor segmentation method that we have developed is composed of two phases: initialization and refinement, as shown in Figure 1. In the first phase, we detect and initially segment the tumor. To perform this operation, the brain is segmented by a combination of histogram analysis, morphological operations and symmetry analysis. Within the brain, the tumor is then detected using a fuzzy classification method or symmetry analysis and some morphological operations. The first method relies on the assumption that the tumor appears in the image with specific gray levels, corresponding to an additional class. The second method relies on the assumption that the brain is roughly symmetrical in shape, and that tumors can be detected as areas that deviate from the symmetry assumption when looking at gray levels. This detection provides the initialization for a more precise segmentation step, performed in the second stage, using a parametric deformable model constrained by fuzzy spatial relations. This allows representing explicitly relations between the tumor and surrounding tissues, thus reinforcing the robustness of the method. 


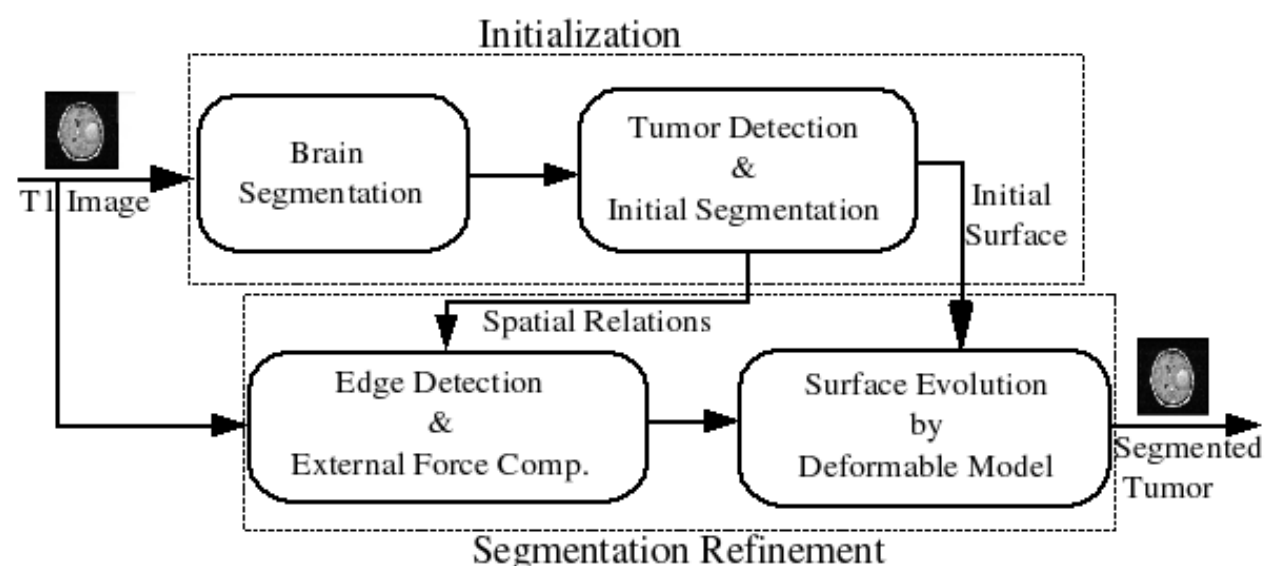

Fig. 1. The segmentation method diagram.

Several sources of imprecision are taken into account in the proposed method. Imprecision is inherently present in the images, due to the observed phenomenon itself (imprecise limits of pathological areas for instance), to the acquisition system and the numerical reconstruction process (leading to spatial and intensity imprecisions). Moreover, available knowledge is also prone to imprecision. For instance we exploit the constant order of the gray levels of the main brain tissues, but the exact range of values of each tissue is imprecise. We will also make use of spatial relations, expressed in linguistic form, such as "near the tumor", which cannot be modeled in a precise way. All these reasons justify the use of fuzzy models in several steps of the proposed approach (fuzzy classification based on gray levels, models of spatial relations).

\section{Computation of the approximate symmetry plane}

Normal human brains possess a high degree of bilateral symmetry although they are not perfectly symmetrical. The symmetry plane of the brain is a good approximation of the mid-sagittal plane, which is defined as the plane that best separates the hemispheres. The automatic detection of this plane in brain images is a useful task and here we will use it to segment the brain and to detect the brain tumors. The computation of the approximate brain symmetry plane is performed according to a method proposed in [32], which is based on the maximization of a symmetry measure. Let us briefly describe it here.

Let $\mathbf{u}$ be a unit vector in $\mathbb{R}^{3}$ and $\Pi_{\mathbf{u}, d}$ a plane in $\mathbb{R}^{3}$ orthogonal to the vector $\mathbf{u}$ and passing at the distance $d$ from the coordinate origin. We denote by $e_{\mathbf{u}, d}(f)$ the reflection of image $f$ with respect to the plane $\Pi_{\mathbf{u}, d}$ : $e_{\mathbf{u}, d}(f)(x, y, z)=f\left(e_{\mathbf{u}, d}(x, y, z)\right)$. An image $f$ is called reflection symmetrical if there exists a reflection plane $\Pi_{\mathbf{u}, d}$ such that $e_{\mathbf{u}, d}(f)=f$. Since there is 
not an exact symmetry in the brain, we consider a degree of symmetry defined as the similarity between $e_{\mathbf{u}, d}(f)$ and $f$ :

$$
\mu_{\mathbf{u}, d}(f)=1-\frac{\left\|f-e_{\mathbf{u}, d}(f)\right\|^{2}}{2\|f\|^{2}} .
$$

The idea is to compute the symmetry measure $\mu_{\mathbf{u}, d}(f)$ of the image $f$ with respect to an arbitrary reflection plane $\Pi_{\mathbf{u}, d}$, and to find the plane leading to the maximal symmetry degree and the corresponding value of symmetry measure $\mu(f)$ :

$$
\mu(f)=\max _{\mathbf{u} \in S^{2}, d \in \mathbb{R}^{+}} \mu_{\mathbf{u}, d}(f) .
$$

First, an initial symmetry plane is estimated based on the ellipsoid of inertia of the image $f$. The three major planes of the ellipsoid of inertia are computed and the plane for which the symmetry measure is maximum is chosen as an initial plane. Then, the orientation and the position of the plane are improved by optimizing in the $3 \mathrm{D}$ space the reflection plane parameters. This leads to an optimum of the proposed similarity measure, and is considered as the approximate symmetry plane.

In the normal brain the symmetry plane of the head in MRI is approximately equal to the symmetry plane of the segmented brain. Although the internal structure of a pathologic brain may depart from its normal bilateral symmetry, the ideal imaginary symmetry plane remains invariant [18]. Therefore in the refinement process of the brain segmentation we can use the symmetry plane of the head instead of the symmetry plane of the segmented brain. In the normal brain, it has also been observed that the symmetry plane of the gray level brain image and the one of the segmented brain are approximately equal. Since pathological brains are usually not symmetric when considering the gray level images, we can compute the symmetry plane of the segmented brain, which exhibits more symmetry and rapidity. Applying this method to images containing tumors provides a good approximation of the mid-sagittal plane, despite the asymmetry induced by the tumors. This is illustrated in Figures $5-$ 9 for a normal brain and for different types of tumors.

\section{Brain segmentation}

The first step of our algorithm consists of brain segmentation. Several methods have been proposed to perform this operation (see e.g. $[19,27,28]$ ) and some of them are available in softwares such as BrainVisa [3], FSL [29] and Brainsuite [26]. Unfortunately most of them fail in the case of the presence of a tumor in the brain, especially if located on the border of the brain (Figure 2). 

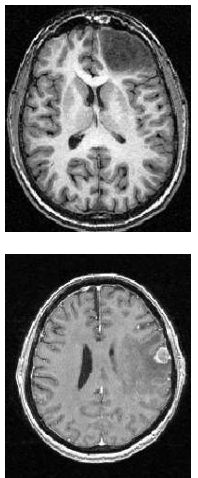

(a)
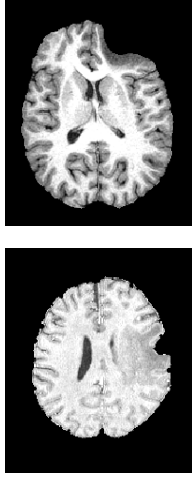

(b)
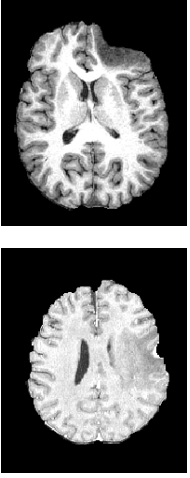

(c)
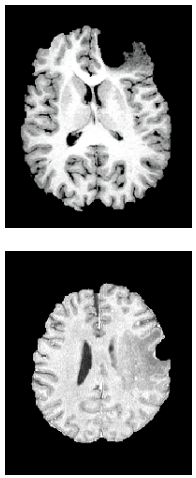

(d)

Fig. 2. Pathological brain segmentation using existing methods. (a) One slice of the original image on two examples. (b) Segmented brain by histogram analysis and morphological operations [19] using BrainVisa [3]. (c) Segmented brain by BET [28] using FSL [29]. (d) Segmented brain by BSE [27] using Brainsuite [26].

To solve this problem, we propose to perform a symmetry analysis, based on the assumption that tumors are generally not symmetrically placed in both hemispheres, while the whole brain is approximately symmetrical.

First we segment the brain using histogram analysis and morphological operations, similarly as in [19]. This leads to a partial segmentation, where a part corresponding to the tumor is missing. The algorithm summarized in Section 3 is applied on the gray level image of the head to compute the approximate symmetry plane, because the segmented brain is not symmetric. The computed symmetry plane on head and segmented brain in normal case are approximately equal and this approximate is acceptable in pathological cases for tumor detection purpose. We then compute the reflected brain with respect to the symmetry plane (Figure 3). By calculating the difference between the reflected brain mask and brain mask in the unsigned 8 bit format (the images have two levels 0 and 255 and after subtraction we select the level 255 ) we obtain an image which contains the removed section of the tumor and other small objects. To select the component which corresponds to the tumor, first we use a morphological opening to disconnect the components. We then select the largest connected component since it corresponds to the removed section of tumor, as confirmed by all our experiments. In the morphological operations the elementary neighborhood corresponds to 6-connectivity. The result can only been considered as an approximation in the tumor area, but it is accurate enough for tumor detection in the next step. Finally we add this result to the segmented brain. The main steps of this method and its results are illustrated on two examples in Figure 3. They correspond to the desired whole brain, including the pathological areas. 


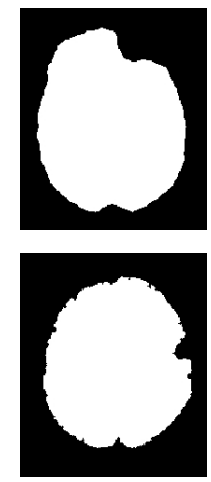

(a)
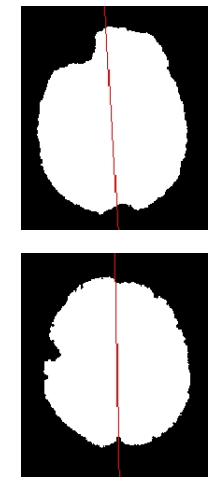

(b)
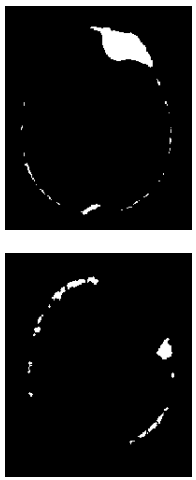

(c)
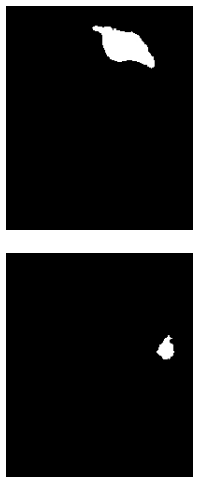

(d)
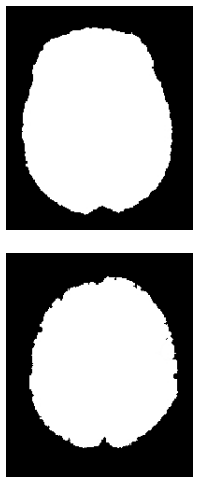

(e)
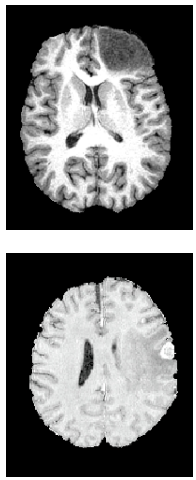

(f)

Fig. 3. The proposed algorithm for pathological brain segmentation (same examples as in Figure 2). (a) Segmented brain by histogram analysis. (b) Reflected brain with respect to the approximate symmetry plane. (c) Difference image of (b) and (a) (bounded difference). (d) Removed section of the tumor obtained by morphological operations from image (c). (e) Final segmented brain. (f) Final gray level segmented brain.

\section{Tumor detection and initial segmentation}

We now described the initial segmentation of the tumor, for which we propose two methods: the first one relies on a fuzzy classification and the second one is based on symmetry analysis.

\subsection{Tumor detection using FPCM}

In [15] we have proposed a new method for hyper-intensity tumor segmentation based on Fuzzy Possibilistic c-Means (FPCM) [22]. FPCM is a combination of Fuzzy c-Means (FCM) and Possibilistic c-Means (PCM) algorithms. In data classification, both membership and typicality are mandatory for data structures interpretation. FPCM computes these two factors simultaneously. FPCM solves the noise sensitivity defect of FCM and overcomes the problem of coincident clusters of PCM. The objective function of FPCM is written as:

$$
J_{m, \eta}(U, T, V ; X)=\sum_{i=1}^{c} \sum_{k=1}^{n}\left(u_{i k}^{m}+t_{i k}^{\eta}\right)\left\|X_{k}-V_{i}\right\|^{2}
$$

where $m>1, \eta<1,0 \leq u_{i k} \leq 1,0 \leq t_{i k} \leq 1, \sum_{i=1}^{c} u_{i k}=1, \forall k, \sum_{k=1}^{n} t_{i k}=$ 1, $\forall i, X_{k}$ denotes the characteristics of a point to be classified (here we use gray levels), $V_{i}$ is the class center, $c$ the number of classes, $n$ the number of points to be classified, $u_{i k}$ the membership of point $X_{k}$ to class $i$, and $t_{i k}$ is the possibilistic typicality value of $X_{k}$ associated with class $i$. 
In order to detect the tumor we use a histogram based FPCM that is faster than the classical FPCM implementation. We classify the extracted brain into five classes, cerebro spinal fluid (CSF), white matter (WM), gray matter (GM), tumor and background (at this study stage we do not consider the edema). To obtain the initial values of the class centers, we use the results of the histogram analysis [19] in the brain extraction step: we define them as the average gray level values of the CSF, WM and GM $\left(\mathbf{m}_{\mathbf{C}}, \mathbf{m}_{\mathbf{W}}\right.$ and $\mathbf{m}_{\mathbf{G}}$, respectively). For the background, the value zero is used. To select the tumor class we assume that the tumor has the highest intensity among the five classes (this is the case for hyper-intensity pathologies such as full-enhanced tumors).

Because of some classification errors, there are undesired additional voxels in the tumor class. To remove these missclassified components, several binary morphological operations are applied to the tumor class. An opening operation is first used to disconnect the components. Then we select the largest connected component, which proved to always correspond to the tumor, even if it has a small size. Here also, the elementary neighborhood of the morphological operations corresponds to 6-connectivity.

We have applied this method to five 3D T1-weighted images with hyperintensity tumors at different locations and with different sizes. In all five cases the tumors have been detected. The results for two images are shown in Figure 4.

Although this method is fully automatic and unsupervised, it is however difficult to generalize to any type of tumors.

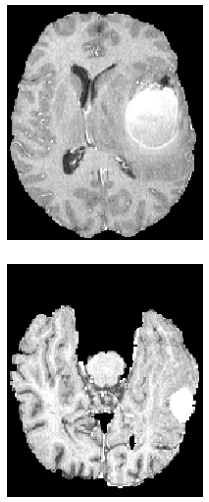

(a)
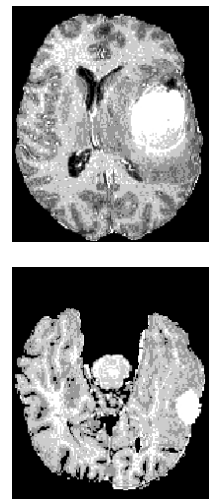

(b)
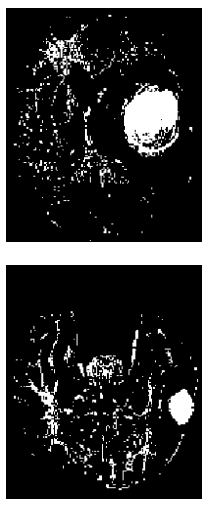

(c)
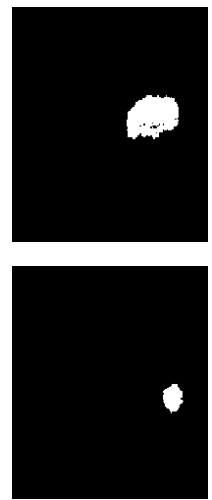

(d)

Fig. 4. Results obtained in the classification step for two 3D images. (a) One axial slice of the segmented brain. (b) Result of FPCM classification. (c) Selected tumor class. (d) Result after morphological operations. 


\subsection{Tumor detection by symmetry analysis}

To overcome the lack of generality of the previous method, we suggest another approach [16], using the approximate symmetry plane. As mentioned in Section 3, since the symmetry plane of the gray level image and the one of the binary mask of the segmented brain in the normal case are approximately equal, to increase the accuracy and to speed-up the algorithm in the pathological case we compute the symmetry plane on the binary mask of the segmented brain (if the symmetry plane has been calculated in the brain segmentation step we use that symmetry plane). Now tumors can be detected by evaluating this asymmetry with respect to the obtained plane. We assume that tumors are localized in only one hemisphere or are not symmetric and the pathological hemisphere (i.e. which includes the largest part of the tumor) is selected manually.

Let $\mathbf{H}_{n}$ denote the histogram of gray levels in the normal hemisphere and $\mathbf{H}_{p}$ the histogram in the pathological hemisphere. The histogram difference $\mathbf{H}_{s}=$ $\mathbf{H}_{p}-\mathbf{H}_{n}$ provides useful information about new intensity classes induced by the tumor. Here we classify the tumors based on their appearance in T1-weighted images with $(\mathrm{T} 1 \mathrm{w})$ or without contrast agent (T1w-CA) into 4 classes:

- non-enhanced tumors, which do not take contrast agent and appear darker than WM in T1w and T1w-CA images (such as low grade glioma (Figure $6)$ ).

- full-enhanced tumors without edema, that take contrast agent and approximately all voxels of the tumor appear hyper-intense (brighter than WM) in T1w-CA images (such as contrast enhanced meningioma without edema Figure 7).

- full-enhanced tumors with edema, where the solid section of the tumor takes contrast agent and appears hyper-intense in $\mathrm{T} 1 \mathrm{w}-\mathrm{CA}$ images and the surrounding edema is darker than GM (such as high grade glioma (Figure 8)).

- ring-enhanced tumors, which have three sections, a central section is the necrosis and appears darker than GM, an enhanced section surrounds the necrosis and appears as hyper-intense and the surrounding edema appears darker than GM in T1w-CA images (such as glioblastoma (Figure 9)).

In the case of a non-enhanced tumor (as in Figure 6) a positive peak can be observed between CSF and GM in $\mathbf{H}_{s}$ that shows the non-enhanced tumor intensity range while in the case of a full-enhanced tumor without edema (as in Figure 7) a positive peak can be observed after the WM peak in $\mathbf{H}_{s}$ that shows the full enhanced tumor intensity range. When a full-enhanced tumor with edema (as in Figure 8) or ring-enhanced tumor with edema (as in Figure 9) exist in the image we have two positive peaks in $\mathbf{H}_{s}$, where the first peak shows the edema intensity range and the second peak shows the 
tumor intensity range, because the intensity of edema is always lower than the intensity of the tumor.

We have considered the peaks with more than 300 voxels, this threshold being based on the analysis of $\mathbf{H}_{s}$ for five healthy brain images of the IBSR database[10] (as in Figure 5 (a)). To extract the tumor we first use a thresholding with tumor peak range values. The gray level ranges of the tumor are selected manually in $\mathbf{H}_{s}$. Some misclassified voxels are removed using morphological operations with the 6-connectivity related elementary neighborhood. First an opening is used to disconnect the components. The largest connected component is then selected since it corresponds to the tumor (as seen in Figures $6,7,8$ and 9 ).

To obtain the tissues around the tumor, we must distinguish two cases: the tumors with edema and the tumors without edema. In the case of a tumor with edema the tissues around the tumor correspond to this edema and it can be extracted by thresholding using the edema gray level range, selected manually in $\mathbf{H}_{s}$. In the case of a tumor without edema the negative peaks observed in $\mathbf{H}_{s}$ correspond to normal tissues, around the tumor, since these tissues are less represented in the hemisphere containing the pathology than in the other hemisphere. These tissues can also be obtained by thresholding (Figures 6, 7, 8 and 9). They will be used for introducing spatial relations in the next section.

We applied this method to 20 cases with different tumor types, at different locations and with different intensities. In all cases it detects and initially segments the tumor (as seen for 7 cases in Figure 13). We have compared the results of the tumor detection process for 4 full-enhanced tumors in Figure 15. These results show better initial segmentation using symmetry analysis method. However, the FPCM method has the advantage of being faster and fully-automatic, while in the symmetry analysis method, the selection of the tumor gray level range in $\mathbf{H}_{s}$ is performed manually.

\section{Segmentation refinement using a deformable model}

The result of tumor segmentation by symmetry analysis and FPCM classification is not accurate enough and therefore we need a method to refine the segmentation. To obtain an accurate segmentation, a parametric deformable method, that has been applied successfully in our previous work to segment internal brain structures [4], is used. 


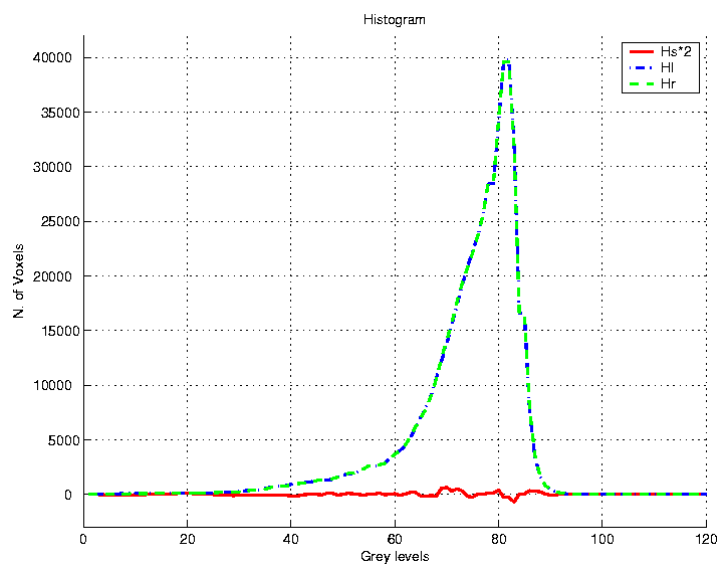

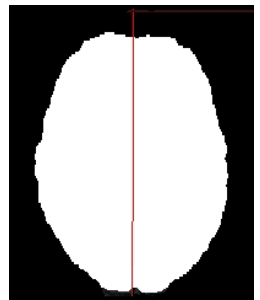

(b)

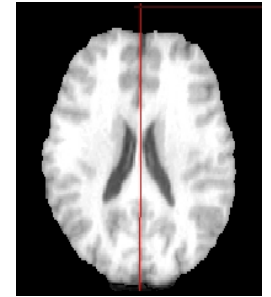

(c)

(a)

Fig. 5. (a) Graph of $\mathbf{H}_{s}, \mathbf{H}_{n}$ and $\mathbf{H}_{p}$ for a normal image (image (c)) (for visualization purposes $\mathbf{H}_{s}$ is multiplied by 2). (b) Symmetry plane superimposed on the brain mask. (c) Symmetry plane superimposed on the segmented brain.

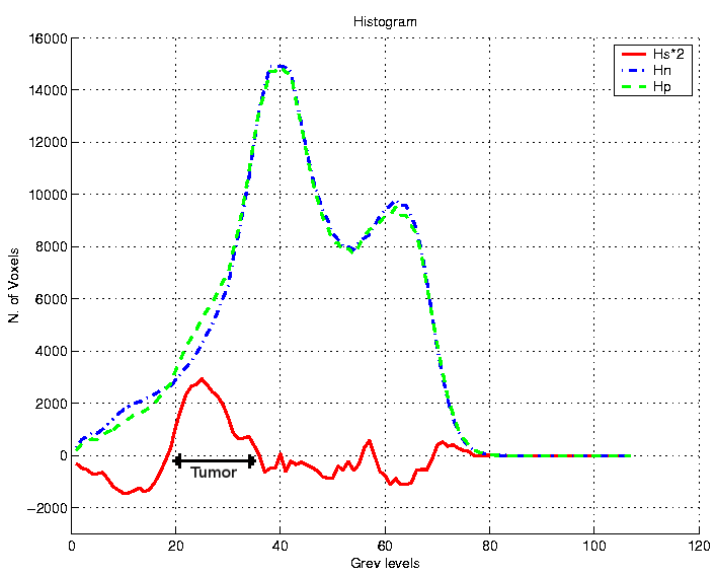

(a)

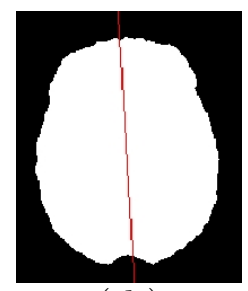

( b)

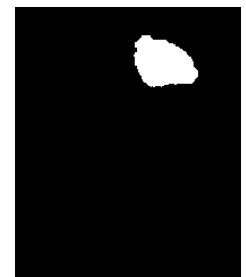

(d)

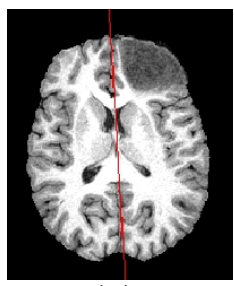

(c)

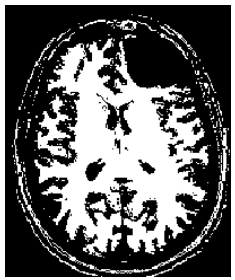

(e)

Fig. 6. (a) Graph of $\mathbf{H}_{s}, \mathbf{H}_{n}$ and $\mathbf{H}_{p}$ for a non-enhanced tumor (image (c)) (for visualization purposes $\mathbf{H}_{s}$ is multiplied by 2). b) Symmetry plane superimposed on the brain mask. (c) Symmetry plane superimposed on the segmented brain. (d) Extracted tumor after morphological operations. (e) Tissues around the tumor.

\subsection{Deformable model}

The segmentation obtained from the previous processing is transformed into a triangulation using an isosurface algorithm [23] based on tetrahedra and is decimated and converted into a simplex mesh, denoted by $\mathbf{X}$ [7].

The evolution of our deformable model is described by the following usual 


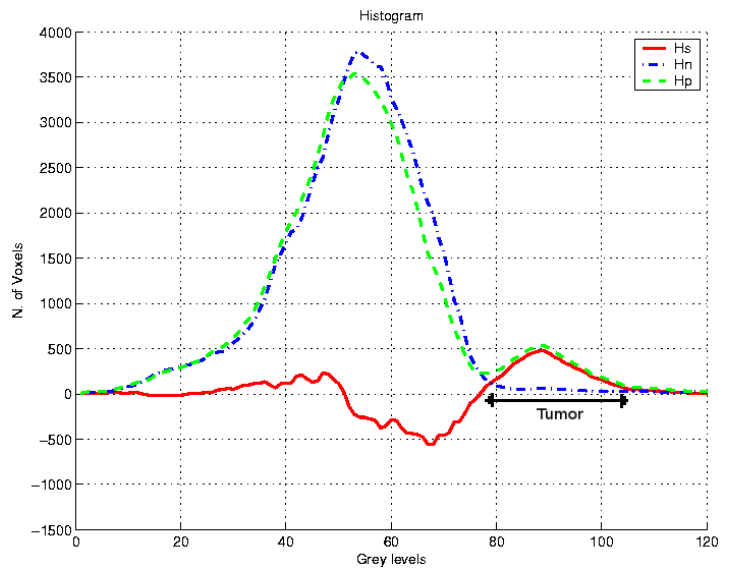

(a)

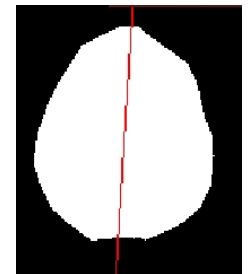

(b)

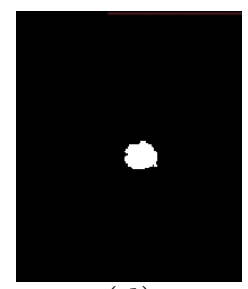

(d)

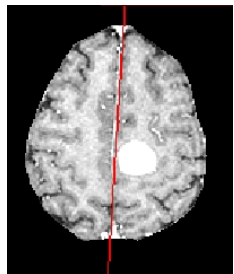

(c)

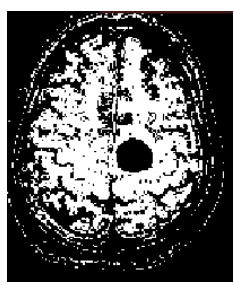

(e)

Fig. 7. (a) Graph of $\mathbf{H}_{s}, \mathbf{H}_{n}$ and $\mathbf{H}_{p}$ for a full-enhanced tumor without edema (image (c)). b) Symmetry plane superimposed on the brain mask. (c) Symmetry plane superimposed on the segmented brain. (d) Extracted tumor after morphological operations. (e) Tissues around the tumor.

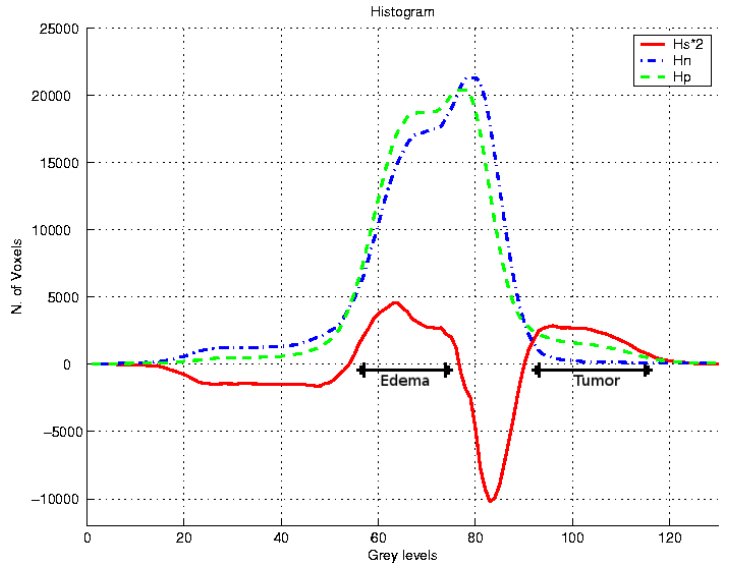

(a)

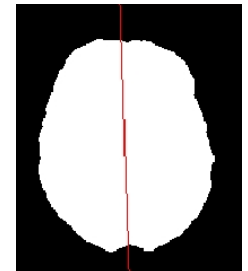

(b)

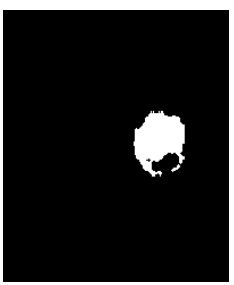

(d)

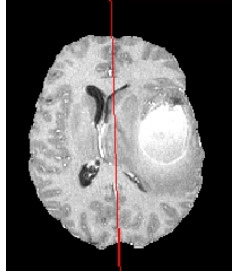

(c)

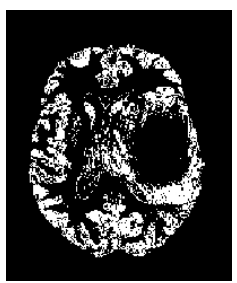

(e)

Fig. 8. (a) Graph of $\mathbf{H}_{s}, \mathbf{H}_{n}$ and $\mathbf{H}_{p}$ for full-enhanced tumor with edema (image (c)) (for visualization purposes $\mathbf{H}_{s}$ is multiplied by 2). b) Symmetry plane superimposed on the brain mask. (c) Symmetry plane superimposed on the segmented brain. (d) Extracted tumor after morphological operations. (e) Tissues around the tumor.

dynamic force equation $[11,34]$ :

$$
\gamma \frac{\partial \mathbf{X}}{\partial t}=\mathbf{F}_{\text {int }}(\mathbf{X})+\mathbf{F}_{\text {ext }}(\mathbf{X})
$$

where $\mathbf{X}$ is the deformable surface, $\mathbf{F}_{\text {int }}$ is the internal force that constrains the regularity of the surface and $\mathbf{F}_{\text {ext }}$ is the external force. In our case, the external force is composed of two terms. The first one is classically derived 


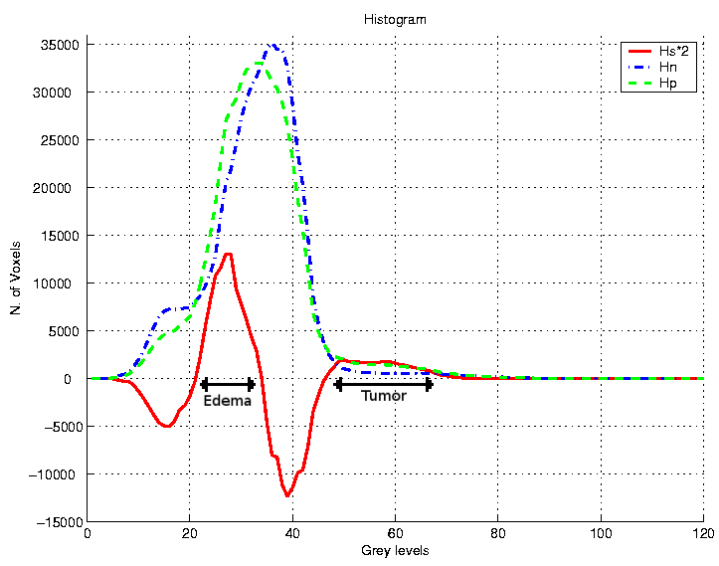

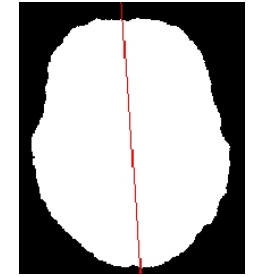

(b)

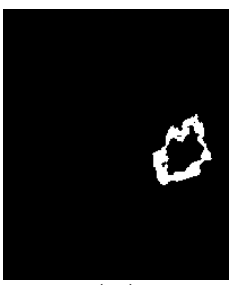

(d)

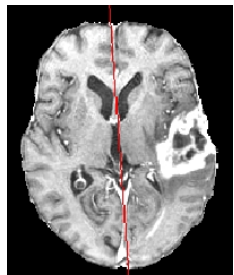

(c)

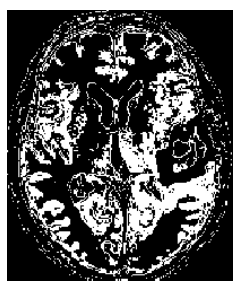

(e)

Fig. 9. (a) Graph of $\mathbf{H}_{s}, \mathbf{H}_{n}$ and $\mathbf{H}_{p}$ for a ring-enhanced tumor with edema (image (c)) (for visualization purposes $\mathbf{H}_{s}$ is multiplied by 2). (b) Symmetry plane superimposed on the brain mask. (c) Symmetry plane superimposed on the segmented brain. (d) Extracted tumor after morphological operations. (e) Tissues around the tumor.

from image edges, and is denoted by $\mathbf{F}_{C}$. It can be written as:

$$
\mathbf{F}_{C}=v(x, y, z)
$$

where $v$ is a Generalized Gradient Vector Flow (GGVF) field introduced by $\mathrm{Xu}$ et al. [35]. A GGVF field $v$ is computed by diffusion of the gradient vector of a given edge map and is defined as the equilibrium solution of the following diffusion equation:

$$
\begin{aligned}
\frac{\partial v}{\partial t} & =g(\|\nabla f\|) \nabla^{2} v-h(\|\nabla f\|)(v-\nabla f) \\
v(x, y, z, 0) & =\nabla f(x, y, z)
\end{aligned}
$$

where $f$ is an edge map and the functions $g$ and $h$ are weighting functions which can be chosen as follows:

$$
\left\{\begin{array}{l}
g(r)=e^{-\frac{r^{2}}{\kappa}} \\
h(r)=1-g(r)
\end{array}\right.
$$

To compute the edge map, we applied the Canny-Deriche edge detector.

The second term of $\mathbf{F}_{\text {ext }}$ is derived from spatial relations and is described next. 


\subsection{Deformable model constrained by spatial relations}

Spatial relations are useful to guide the recognition of objects in images since they provide an important information about the spatial organization of these objects. Two main classes of spatial relations can be considered: topological relations, such as inclusion, exclusion and adjacency, and metric relations such as distances and orientations. Here we use a combination of topological and distance information. The evolution process of the deformable model can be guided by a combination of such relations, via information fusion tools.

In the case of tumor detection by symmetry analysis, two types of information are available: the initial detection and the surrounding tissues. Therefore we use (i) the distance from the initial segmented tumor, and (ii) the tissues around the tumor which were obtained in the previous step (as in Figure 11). The idea is that the contour of the tumor should be situated somewhere in between the boundary of the initial detection and the boundary of the tumor around tissues (excluding the background). This constraint also prevents the deformable model from leakage in the weak boundaries.

For distance relations such as "near the initial segmented tumor", we define a fuzzy interval $f$ of trapezoidal shape on the set of distances $\mathbb{R}^{+}$(Figure 10). The kernel of $f$ is defined as $\left[0, n_{1}\right]$ and its support as $\left[n_{1}, n_{2}\right]$. Here $n_{1}$ and $n_{2}$ are defined according to the largest distance between the initial segmentation of the tumor and its surrounding tissues. To obtain a fuzzy subset of the image space, $f$ is combined with a distance map $d_{A}$ to the reference object $A$ : $d(P)=f\left(d_{A}(P)\right)$ where $P$ is a point of the space.

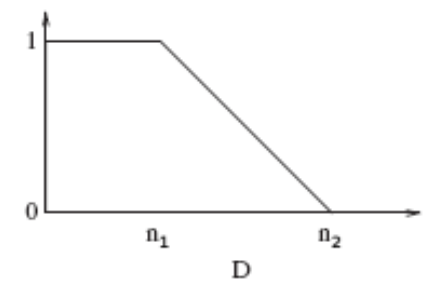

Fig. 10. Fuzzy interval on the set of distances corresponding to the relation "near".

The relation "near the tissues surrounding the tumor" is modeled in a similar way. These two relations are represented as fuzzy sets in the image space (as shown in Figure 11).

These relations are combined using a conjunctive fusion operator (a t-norm such as minimum), leading to a fuzzy set $\mu_{R}$. The resulting fuzzy set provides high values in the region where both relations are satisfied, and lower elsewhere. As shown in Figure 11, this result is a good region of interest for the contour to be detected. 


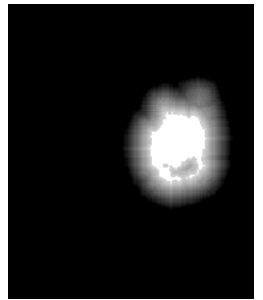

(a)

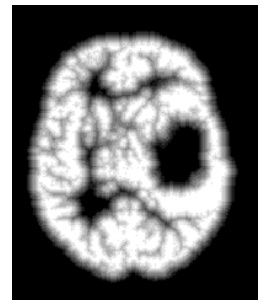

(b)

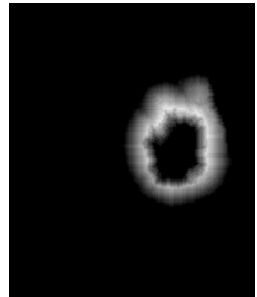

(c)

Fig. 11. Spatial relations used for segmenting the tumor detected in Figure 8 (highest gray level values correspond to regions where the spatial relation is best satisfied). (a) Near the tumor. (b) Relation provided by the tissues surrounding the tumor. (c) Fusion of the two relations.

In [4], several methods to compute the force from a fuzzy set $\mu_{R}$ were proposed. For instance, if $\mu_{R}(x)$ denotes the degree of satisfaction of the fuzzy relation at point $x$, and $\operatorname{supp}(R)$ the support of $\mu_{R}$, then we can derive the following potential:

$$
P_{R}(x)=1-\mu_{R}(x)+d_{\operatorname{supp}(R)}(x),
$$

where $d_{\operatorname{supp}(R)}$ is the distance to the support of $\mu_{R}$, used to have a non-zero force outside the support. The force $\mathbf{F}_{R}$ associated with the potential $P_{R}$ is derived as follows:

$$
\mathbf{F}_{R}(x)=-\left(1-\mu_{R}(x)\right) \frac{\nabla P_{R}(x)}{\left\|\nabla P_{R}(x)\right\|}
$$

This force is combined to the classical external force derived from edge information $\mathbf{F}_{C}$ :

$$
\mathbf{F}_{\text {ext }}=\lambda \mathbf{F}_{C}+\nu \mathbf{F}_{R}
$$

where $\lambda$ and $\nu$ are weighting coefficients. The role of $\mathbf{F}_{R}$ is to force the deformable model to stay within regions where specific spatial relations are fulfilled. Figure 12 shows examples of two spatial relations and their corresponding forces.

\section{$7 \quad$ Results and validation}

We have applied the proposed method to MR data from 20 patients with cerebral tumors. Images were acquired on a $1.5 \mathrm{~T}$ (General Electric Medical System) scanner using an axial 3D IR-SPGR T1-weighted sequence with or without contrast agent. The volume dimension is $256 \times 256 \times 124$ and the voxel size is typically $1 \times 1 \times 1.3 \mathrm{~mm}^{3}$. These images contain tumors with different sizes, intensities, shapes and locations. This allows us to illustrate the large field of application of our method. 


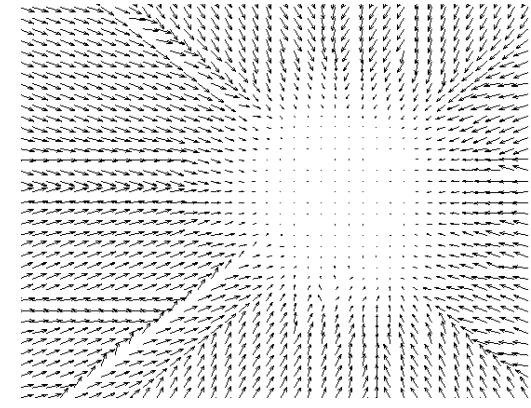

(a)

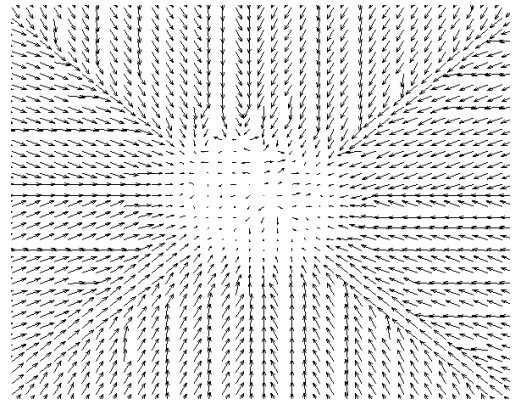

(b)

Fig. 12. External force $\mathbf{F}_{R}$ computed from a fuzzy subset $\mu_{R}$ corresponding to a spatial relation $R$. (a) The force $\mathbf{F}_{R}$ computed from $\mu_{R}$ for the relation "near the tumor" in Figure 11(a). (b) The force computed from the fusion of the two relations of Figure 11(c) (for visualization purposes an under-sampling has been performed).

The segmentation results for 7 cases with initial segmentation by symmetry analysis and refinement by constrained deformable model are shown in Figures 13 and 14 . The results can be compared with manually segmented tumors. The 4 first cases are full-enhanced and ring-enhanced tumors while the 3 last tumors are non-enhanced tumors. In all cases, the initial detection based on symmetry analysis only provides a part of the tumor. The whole tumor is successfully recovered by the second segmentation step using the deformable model and the spatial relations.

The evaluation of the segmentation results was performed through a quantitative comparison with the results of a manual segmentation. Let us denote by $M$ the manually segmented tumor and $A$ the segmented tumor by our method. We used five measures to evaluate the results which are:

- ratio of correct detection: $T_{p}=\frac{N_{T p}}{N_{M}} * 100 \%$, where $N_{T p}$ is the number of true positive voxels and $N_{M}$ is the cardinality of $M$;

- ratio of false detection: $F_{p}=\frac{N_{F p}}{N_{A}} * 100 \%$, where $N_{F p}$ is the number of false positive and $N_{A}$ is the cardinality of $A$;

- similarity index: $S=\frac{2 N_{T p}}{N_{M}+N_{A}} * 100 \%$;

- Hausdorff distance between $A$ and $M$, defined as $D_{H}=$ $\max (h(M, A), h(A, M))$ where $h(M, A)=\max _{m \in M} \min _{a \in A} d(m, a)$, and $d(m, a)$ denotes the Euclidean distance between $m$ and $a$ ( $m$ and $a$ are points of $M$ and $A$ respectively);

- average distance $\left(D_{m}\right)$ between the surfaces of $M$ and $A$.

The $T_{p}$ value indicates how much of the actual tumor has been correctly detected, while $F_{p}$ indicates how much of the detected tumor is wrong. The similarity index $S$ is more sensitive to differences in location. For example, if region $A$ completely includes region $M$, while $M$ is one half of $A$, then the $T_{p}$ value is $100 \%$ while the $S$ value is $67 \%$. Since usually most errors are located at the boundary of the segmented regions, small regions will have smaller $S$ 
and $T_{p}$ values than large regions. Therefore we also use the average distance and the Hausdorff distance that do not depend on the region size.

The quantitative results obtained by comparing the automatic segmentations with the available manual segmentations are provided in Table 1 for 20 cases. In Table 1, where the tumor size varies from $2365 \mathrm{~mm}^{3}$ to $817997 \mathrm{~mm}^{3}$ with 3 types of appearance in intensity: full-enhanced, ring-enhanced and nonenhanced. The similarity index varies from $86 \%$ to $96 \%$ with a mean of $92 \%$ and the correct detection ratio varies from $79 \%$ to $97 \%$ with a mean of $93 \%$ that shows a high matching between the manually and automatic segmented tumor. The false detection ratio ranges between $2 \%$ and $12 \%$ with an average of $7.92 \%$, which shows a good accuracy of segmentation (since it is normalized by $N_{A}$ ). The Hausdorff distance, that is a maximum distance and therefore a particularly severe evaluation, varies from $1.5 \mathrm{~mm}$ to $8.12 \mathrm{~mm}$ with a mean of $4.57 \mathrm{~mm}$. This expresses a good position of the boundary of the tumors and we believe that it is due to the use of spatial relations to constrain the deformable model. The mean value of the average distance is $0.69 \mathrm{~mm}$ that is approximately equal to the half of the voxel size, and constitutes a very good result.

The results of this table also show that the quality of the segmention for ring-enhanced and full-enhanced tumors is better than the non-enhanced tumors because of their well-defined boundaries. Improvement of the method for segmenting non-enhanced tumors could still be useful.

The average computation time for detection and segmentation of each tumor by the symmetry analysis method, in 20 cases, is about 4.5 minutes, in addition to 1 minute for manual operation to select the tumor gray levels rang in $H_{s}$, while for FPCM method the computation time is about 3.5 minutes.

The segmentation results based on the two proposed methods for initialization were compared on 4 full-enhanced cases. The results are provided in Table 2. They show that using the symmetry analysis and constrained deformable model improves the segmentation quality. But on the other hand the FPCM method is unsupervised and faster than the symmetry analysis method.

\section{Conclusion}

We have developed a hybrid segmentation method that uses both region and boundary information of the image to segment the tumor. We compared a fuzzy classification method and a symmetry analysis method to detect the tumors and we have used a deformable model constrained by spatial relations 


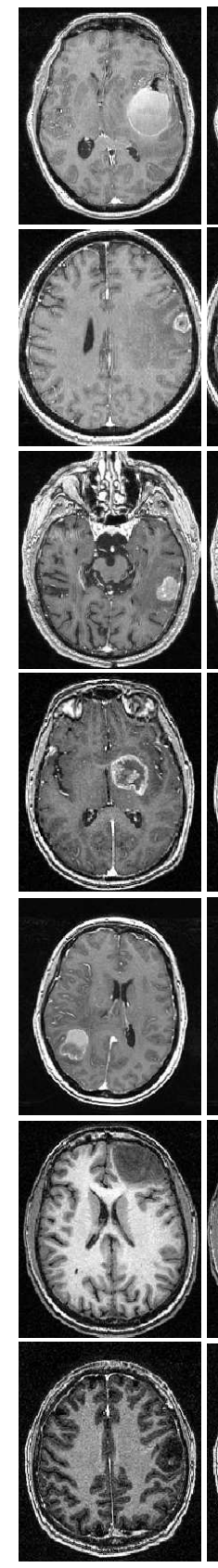

(a)
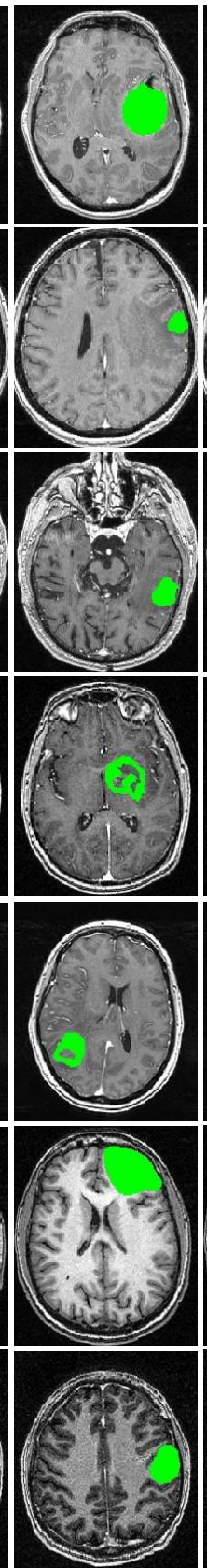

(b)
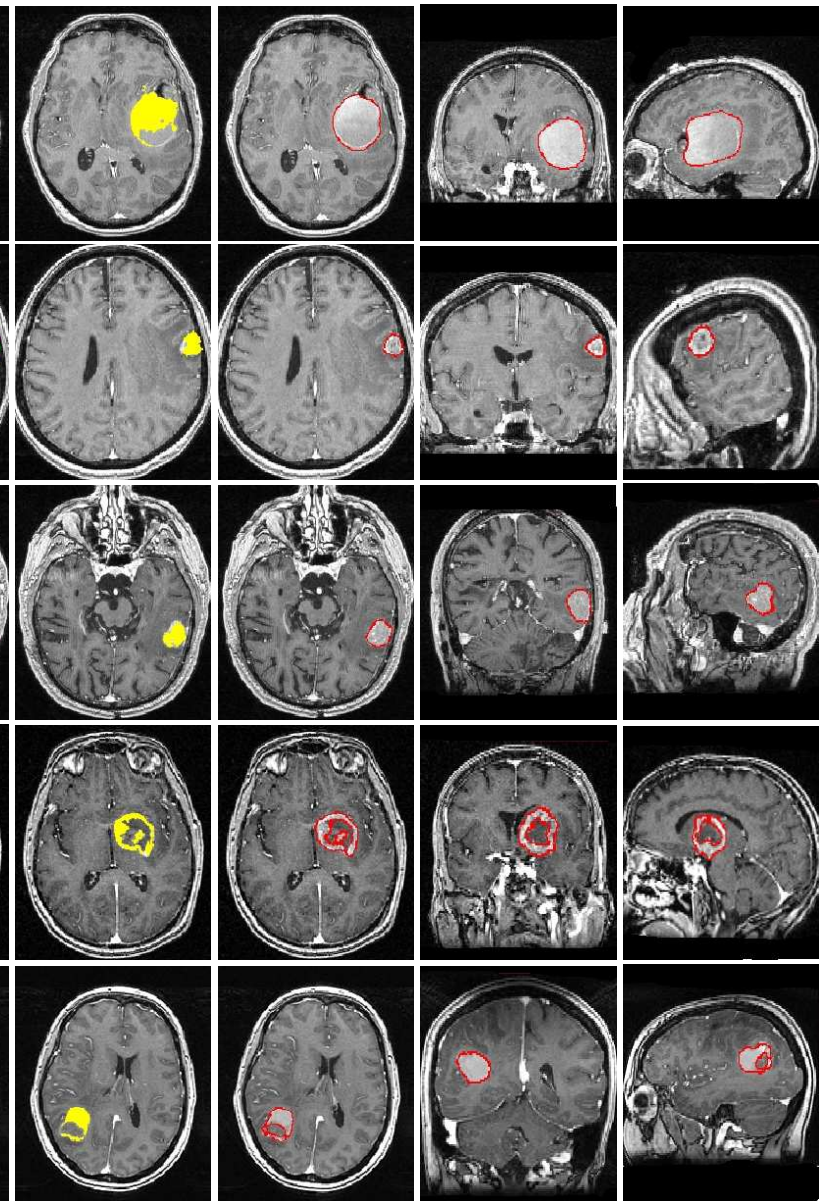

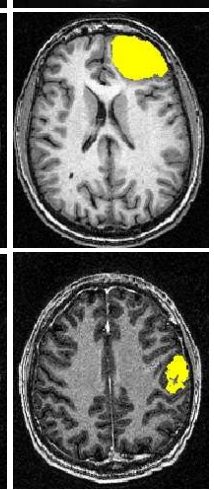

(c)

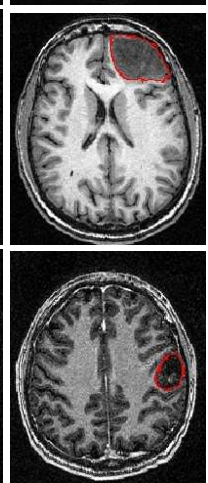

(d)

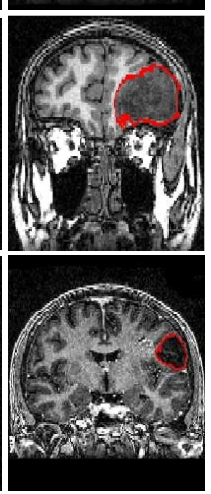

(e)

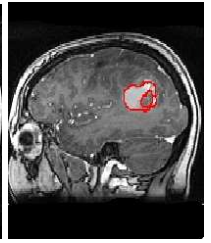

Fig. 13. Comparison of manual and automatic segmentation results using symmetry analysis and constrained deformable model for 7 different cases. (a) One axial slice of the original image. (b) Manual segmentation superimposed on the axial slice. (c) Initial detection by symmetry analysis. (d) Final segmentation. (e) Result superimposed on a coronal slice. (f) Result superimposed on a sagittal slice.

for segmentation refinement. This work shows that the symmetry plane is a useful feature for tumor detection. In comparison with other methods, our approach has some advantages such as automation (in the symmetry analysis method, a reduced interaction is required to select the appropriate peaks in 


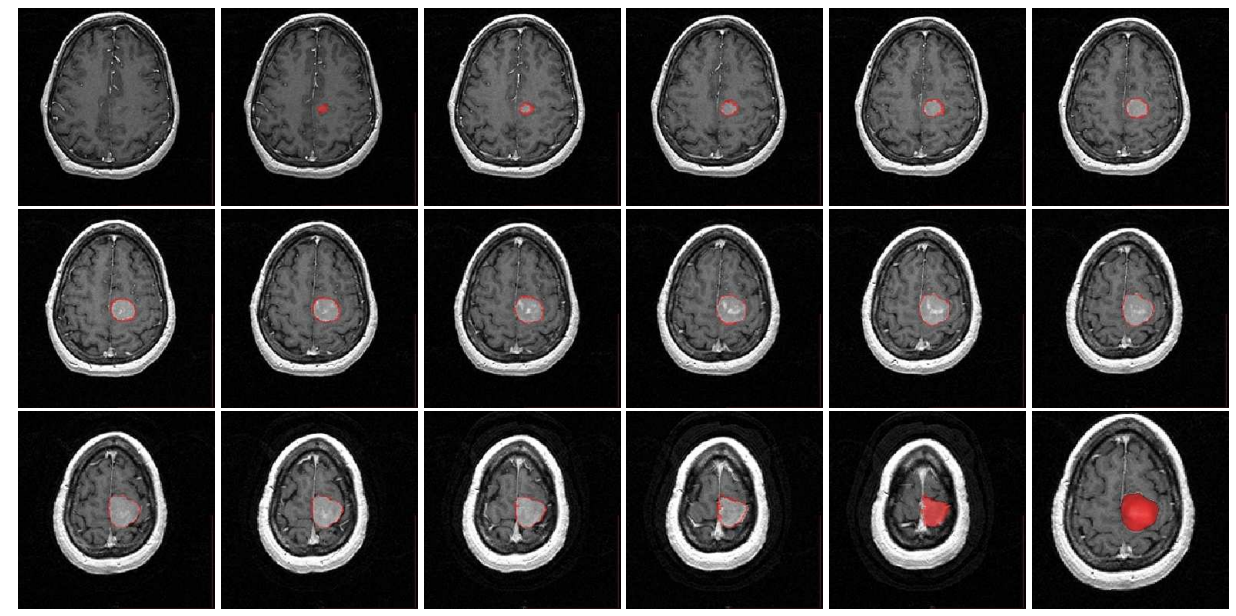

Fig. 14. All slices of a segmented tumor. The last image (bottom-right) is the 3D view of the segmented tumor.

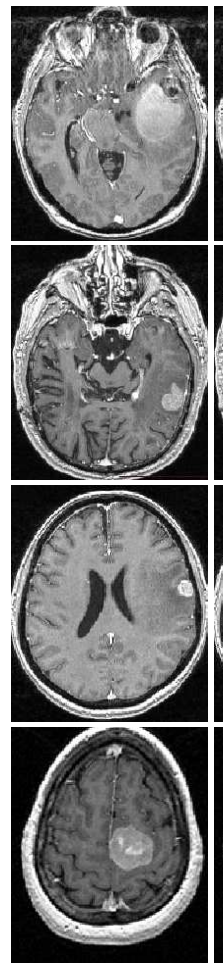

(a)

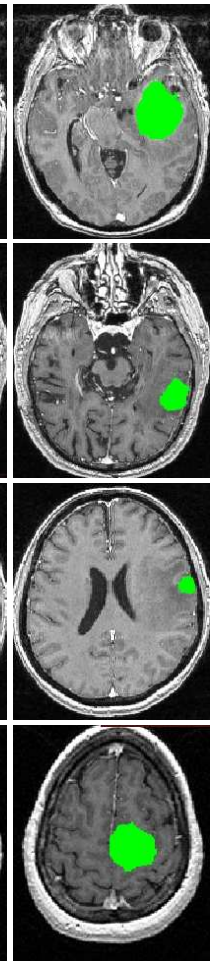

(b)

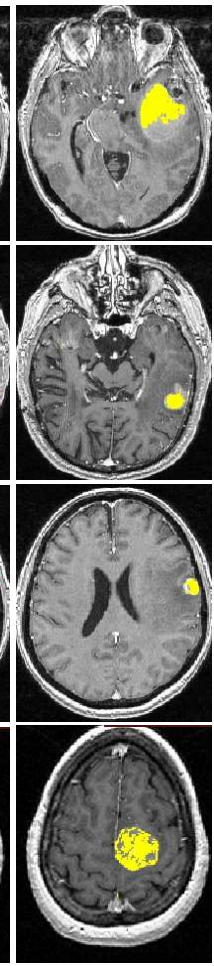

(c)

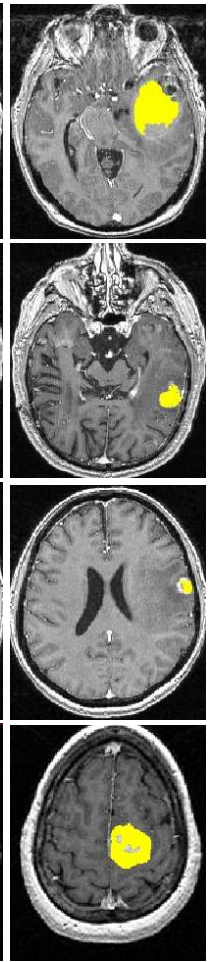

(d)

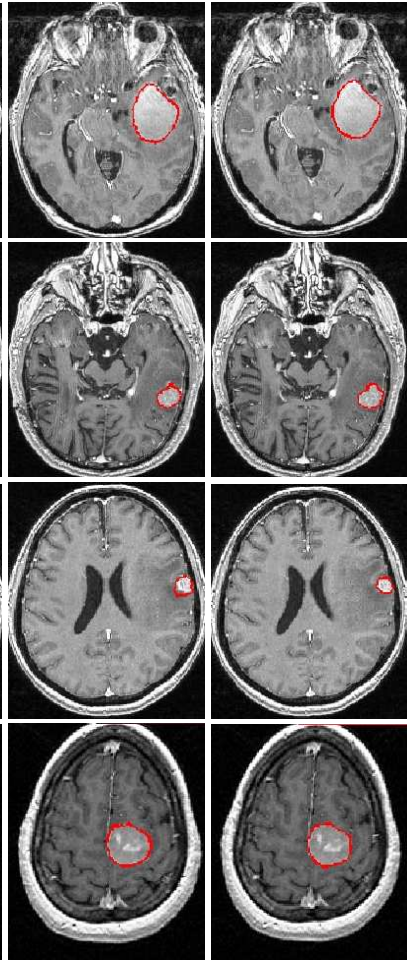

(e)

(f)

Fig. 15. Comparison of FPCM method with manual and symmetry analysis segmentation results. (a) One axial slice of the original image. (b) Manual segmentation. (c) Initial detection by FPCM. (d) Initial detection by symmetry analysis. (e) Final result by FPCM method and deformable model. (f) Final result by symmetry analysis and spatial relation constrained deformable model.

the difference histogram), more generality with respect to the wide range of tumors. We also anticipate that it is applicable to any type of image such as T2weighted, FLAIR, etc. Unfortunately there is not a gold standard to compare quantitatively the method with existing methods. In comparison with recent 


\begin{tabular}{|ccccccc|}
\hline Tumor & $M$ & $S_{i}$ & $T_{p}$ & $F_{p}$ & $D_{H}$ & $D_{m}$ \\
type & $m m^{3}$ & $\%$ & $\%$ & $\%$ & $m m$ & $m m$ \\
\hline FE1 & 9822.4 & 92 & 92 & 7.53 & 4.19 & 0.56 \\
FE2 & 62406.0 & 96 & 97 & 4.38 & 4.63 & 0.62 \\
FE3 & 2312.2 & 90 & 91 & 11.40 & 4.26 & 0.49 \\
FE4 & 11118.0 & 94 & 91 & 2.01 & 4.89 & 0.47 \\
FE5 & 5776.16 & 91 & 95 & 12.59 & 3.97 & 0.45 \\
RE1 & 13213.2 & 92 & 96 & 12.11 & 3.35 & 0.63 \\
RE2 & 23787.2 & 92 & 94 & 10.18 & 3.57 & 0.50 \\
RE3 & 42320.4 & 95 & 96 & 6.49 & 3.36 & 0.35 \\
RE4 & 42322.9 & 89 & 86 & 8.33 & 7.53 & 1.04 \\
NE1 & 14967.1 & 96 & 97 & 5.44 & 2.00 & 0.38 \\
NE2 & 9543.9 & 89 & 88 & 9.85 & 5.89 & 1.01 \\
NE3 & 63611.4 & 95 & 95 & 5.09 & 5.01 & 0.62 \\
NE4 & 34396.4 & 95 & 94 & 3.84 & 1.50 & 1.27 \\
NE5 & 61056.6 & 86 & 79 & 6.71 & 8.12 & 1.68 \\
NE6 & 15349.0 & 95 & 97 & 7.35 & 3.32 & 0.40 \\
NE7 & 21621.7 & 93 & 96 & 9.92 & 4.45 & 0.64 \\
NE8 & 16184.0 & 89 & 88 & 11.02 & 6.15 & 0.99 \\
NE9 & 5978.9 & 90 & 92 & 12.11 & 3.49 & 0.50 \\
NE10 & 69748.4 & 95 & 96 & 4.75 & 6.71 & 0.51 \\
NE11 & 51857.6 & 94 & 96 & 7.28 & 4.92 & 0.64 \\
\hline Average & 68869.7 & 92 & 93 & 7.92 & 4.57 & 0.69 \\
\hline
\end{tabular}

Table 1

Evaluation of the segmentation results of tumors by symmetry analysis and constrained deformable model on a few 3D MR images for which a manual segmentation was available ( $M$ denotes the manually segmented tumor and $\mathrm{FE}, \mathrm{RE}$ and NE denote full-enhanced, ring-enhanced and non-enhanced cases, respectively).

works such as Dou et el. [8], Corso et al. [5] and Prastawa et al. [24], where a quantitative evaluation has been done, our results based on similarity index and correct detection ratio are better than or equal to the ones reported in these works. 


\begin{tabular}{|cccccccc|}
\hline Tumor & Method & $M$ & $S_{i}$ & $T_{p}$ & $F_{p}$ & $D_{H}$ & $D_{m}$ \\
type & & $m m^{3}$ & $\%$ & $\%$ & $\%$ & $m m$ & $m m$ \\
\hline FE1 & FPCM & 9822.4 & 89 & 82 & 1.92 & 4.60 & 0.68 \\
FE1 & Symmetry & 9822.4 & 92 & 92 & 7.53 & 4.19 & 0.56 \\
FE2 & FPCM & 62406.0 & 92 & 93 & 9.57 & 6.53 & 1.10 \\
FE2 & Symmetry & 62406.0 & 96 & 97 & 4.38 & 4.63 & 0.62 \\
FE3 & FPCM & 2312.2 & 86 & 85 & 11.82 & 4.17 & 0.64 \\
FE3 & Symmetry & 2312.2 & 90 & 91 & 11.40 & 4.26 & 0.49 \\
FE4 & FPCM & 11118.0 & 94 & 97 & 9.31 & 5.09 & 0.62 \\
FE4 & Symmetry & 11118.0 & 94 & 91 & 2.01 & 4.89 & 0.47 \\
\hline Average & FPCM & 21414.6 & 90 & 89 & 8.15 & 5.10 & 0.76 \\
Average & symmetry & 21414.6 & 93 & 93 & 6.33 & 4.49 & 0.54 \\
\hline
\end{tabular}

Table 2

Evaluation of the segmentation results of full-enhanced tumors by FPCM and deformable model method on four 3D MR images for which a manual segmentation was available.

A limit of our approach is that the symmetry analysis may fail in the case of a symmetrical tumor across the mid-sagittal plane. However this case is very rare.

Future work aims at determining the type of tumor based on an ontology of tumors. Further segmentation, such as segmentation of the edema, would be useful for this aim. Using other features of tumors such as texture information can be useful in improving the results of detection and segmentation.

Our results can also serve as a preliminary step for segmenting surrounding structures by using fuzzy spatial relations defined according to the type of the tumors, as shown in $[13,14]$. They can also be incorporated in longitudinal studies, for analyzing the evolution of the tumors and their impact on surrounding structures, can be used for diagnosis, treatment planning, terapeutical monitoring, surgery and pathological brain modeling.

\section{Acknowledgements}

Hassan Khotanlou is supported by Bu-Ali Sina University. This work was partly supported by grants from ParisTech - Région Ile de France, GET and 
ANR, during the post-doctoral work of O. Colliot and J. Atif at ENST.

We would like to thank Professor Desgeorges at Val-de-Grâce hospital, Professor Devaux at Sainte-Anne Hospital, Professor Carpentier at Laribosière Hospital and Professor Duffau at Salpétrière Hospital for providing the images and their medical expertise. The image of Figure 14 and the last image in Figure 15 was provided by the Center for Morphometric Analysis at Massachusetts General Hospital and is available at http://www.cma.mgh.harvard.edu/ibsr/.

\section{References}

[1] A. S. Capelle, O. Colot, and C. Fernandez-Maloigne. Evidential segmentation scheme of multi-echo MR images for the detection of brain tumors using neighborhood information. Information Fusion, 5:203-216, 2004.

[2] M.C. Clark, L.O. Lawrence, D.B. Golgof, R. Velthuizen, F.R. Murtagh, and M.S. Silbiger. Automatic tumor segmentation using knowledge-based techniques. IEEE Transactions on Medical Imaging, 17(2):187-201, April 1998.

[3] Y. Cointepas, J.-F. Mangin, L. Garnero, J.-B. Poline, and H. Benali. BrainVISA: Software platform for visualization and analysis of multi-modality brain data. Neuroimage, 13(6):S98, 2001.

[4] O. Colliot, O. Camara, and I. Bloch. Integration of Fuzzy Spatial Relations in Deformable Models - Application to Brain MRI Segmentation. Pattern Recognition, 39:1401-1414, 2006.

[5] J.J. Corso, E. Sharon, and A. Yuille. Multilevel segmentation and integrated Bayesian model classification with an application to brain tumor segmentation. In MICCAI2006, volume LNCS4191, pages 790-798, Copenhagen, Denmark, Oct. 2006.

[6] M. B. Cuadra, C. Pollo, A. Bardera, O. Cuisenaire, J. Villemure, and J.-P. Thiran. Atlas-based segmentation of pathological MR brain images using a model of lesion growth. IEEE Transactions on Medical Imaging, 23(10):13011313, October 2004.

[7] H. Delingette. General object reconstruction based on simplex meshes. IJCV, 32(2):111-146, 1999.

[8] W. Dou, S. Ruan, Y. Chen, D. Bloyet, and J.M. Constans. A framework of fuzzy information fusion for segmentation of brain tumor tissues on MR images. Image and Vision Computing, 25:164-171, 2007.

[9] S. Ho, E. Bullitt, and G. Gerig. Level set evolution with region competition: Automatic 3D segmentation of brain tumors. In ICPR, pages 532-535, Quebec, August 2002. 
[10] IBSR. Internet Brain Segmentation Repository, Center for Morphometric Analysis at Massachusetts General Hospital, www.cma.mgh.harvard.edu/ibsr/.

[11] M. Kass, A. Witkin, and D. Terzopoulos. Snakes: Active contour models. International Journal of Computer Vision, 1(4):321-331, January 1988.

[12] M.R. Kaus, S.K. Warfield, A. Nabavi, E. Chatzidakis, P.M. Black, F.A. Jolesz, and R. Kikinis. Segmentation of meningiomas and low grade gliomas in MRI. In MICCAI, volume LNCS 1679, pages 1-10, Cambridge UK, September 1999.

[13] H. Khotanlou, J. Atif, E. Angelini, H. Duffau, and I. Bloch. Adaptive Segmentation of Internal Brain Structures in Pathological MR Images Depending on Tumor Types. In ISBI 2007, pages 588-591, Washington DC, USA, apr 2007.

[14] H. Khotanlou, J. Atif, B. Batrancourt, O. Colliot, E. Angelini, and I. Bloch. Segmentation de tumeurs cérébrales et intégration dans un modèle de l'anatomie. In Reconnaissance des Formes et Intelligence Artificielle, RFIA'06, Tours, France, jan 2006.

[15] H. Khotanlou, J. Atif, O. Colliot, and I. Bloch. 3D Brain Tumor Segmentation Using Fuzzy Classification and Deformable Models. In WILF, volume LNCS 3849, pages 312-318, Crema, Italy, sep 2005.

[16] H. Khotanlou, O. Colliot, and I. Bloch. Automatic Brain Tumor Segmentation using Symmetry Analysis and Deformable Models. In International Conference on Advances in Pattern Recognition ICAPR, Kolkata, India, jan 2007.

[17] A. Lefohn, J. Cates, and R. Whitaker. Interactive, GPU-based level sets for 3D brain tumor segmentation. Technical report, University of Utah, April 2003.

[18] Y. Liu, R. T. Collins, and W. E. Rothfus. Automatic extraction of the central symmetry (mid-sagittal) plane from neuroradiology images. Technical report, Carnegie Mellon Univ., Pittsburgh, PA, The Robotics Institute, 1996.

[19] J.-F. Mangin, O. Coulon, and V. Frouin. Robust brain segmentation using histogram scale-space analysis and mathematical morphology. In MICCAI, pages 1230-1241, Cambridge USA, October 1998.

[20] N. Moon, E. Bullitt, K.V. Leemput, and G. Gerig. Model-based brain and tumor segmentation. In ICPR, pages 528-531, Quebec, August 2002.

[21] G. Moonis, J. Liu, J. K. Udupa, and D. B. Hackney. Estimation of tumor volume with fuzzy-connectedness segmentation of MR images. American Journal of Neuroradiology, 23:352-363, 2002.

[22] N.R. Pal, K. Pal, and J.C Bezdek. A mixed c-mean clustering model. In IEEE International Conference on Fuzzy Systems, volume 1, pages 11-21, July 1997.

[23] B. Piquet, C.T. Silva, and A. Kaufman. Tetra-cubes: an algorithm to generate 3D isosurfaces based upon tetrahedra. In Brazilian Symposium on Computer Graphics and Image Processing, SIBGRAPI 96, volume 21, pages 205-210, 1996. 
[24] M. Prastawa, E. Bullitt, S. Ho, and G. Gerig. A brain tumor segmentation framework based on outlier detection. Medical Image Analysis, 18(3):217-231, 2004 .

[25] M. Schmidt, I. Levner, R. Greiner, A. Murtha, and A. Bistritz. Segmenting Brain Tumors using Alignment-Based Features. In IEEE International Conference on Machine learning and Applications, pages 215-220, 2005.

[26] D.W. Shattuck and R.M. Leahy. BrainSuite: an automated cortical surface identification tool. Medical Image Analysis, 6(2):129-142, 2002.

[27] D.W. Shattuck, S.R. Sandor-Leahy, K.A. Schaper, D.A. Rottenberg, and R.M. Leahy. Magnetic resonance image tissue classification using a partial volume model. NeuroImage, 13(5):856-876, 2001.

[28] S.M. Smith. Fast robust automated brain extraction. Human Brain Mapping, 17(3):143-155, 2002.

[29] S.M. Smith, P.R. Bannister, C.F. Beckmann, J.M. Brady, S. Clare, D. Flitney, P. Hansen nad M. Jenkinson, D. Leibovici, B. Ripley, M.W. Woolrich, and Y. Zhang. FSL: new tools for functional and structural brain image analysis. In Seventh International Conference on Functional Mapping of the Human Brain, volume NeuroImage, 13, Brighton, UK, June 2001.

[30] J-P. Thirion. Image matching as a diffusion process: an analogy with Maxwells demons. Medical Image Analysis, 2(3):243-260, 1998.

[31] AW Toga, P.M. Thompson, M.S. Mega, K.L. Narr, and R.E. Blanton. Probabilistic approaches for atlasing normal and disease-specific brain variability. Anatomy and Embryology, 204(4):267-282, 2001.

[32] A. Tuzikov, O. Colliot, and I. Bloch. Evaluation of the symmetry plane in 3D MR brain images. Pattern Recognition Letters, 24(14):2219-2233, oct 2003.

[33] K. Xie, J. Yang, Z.G. Zhang, and Y.M. Zhu. Semi-automated brain tumor and edema segmentation using MRI. European Journal of Radiology, 56:12-19, 2005 .

[34] C. Xu, D. Pham, and J. Prince. Medical image segmentation using deformable models. In J. Fitzpatrick and M. Sonka, editors, Handbook of Medical Imaging, volume 2, pages 129-174. SPIE Press, 2000.

[35] C. Xu and J.L. Prince. Snakes, shapes and gradient vector flow. IEEE Transactions on Image Processing, 7(3):359-369, 1998.

[36] J. Zhou, K.L. Chan, V.F.H Chong, and S.M. Krishnan. Extraction of brain tumor from MR images using one-class support vector machine. In IEEE Conference on Engineering in Medicine and Biology, pages 6411-6414, 2005.

[37] Y. Zhu and H. Yang. Computerized tumor boundary detection using a Hopfield neural network. IEEE Transactions on Medical Imaging, 16(1):55-67, 1997. 